\title{
Endotoxins: Structure, Function and Recognition
}

\author{
(X. Wang and P. Quinn, eds., Series: Subcellular Biochemistry, \\ Vol. 53, Springer, 2010, 415 p., \$239)
}

DOI: $10.1134 / \mathrm{S} 0006297911050129$

The book consists of two parts that include 17 chapters prepared by an international team of well-known experts. In Part I consisting of eight chapters, structure and properties of endotoxins are considered. Chapters 13 highlight characterization of lipopolysaccharides (LPS) of Gram-negative bacteria, their purification, and the relationship between their structure, function, and activity. Chapters 4-6 deal with the diversity of core oligosaccharide in LPS, Salmonella-regulated LPS modifications, and variation of O-antigens in Gram-negative bacteria. Chapters 7 and 8 contain data on regulators of TLR4 signaling by endotoxins and membrane partitioning.

Part II includes nine chapters, which highlight infection, treatment, and immunity topics. Chapter 9 deals with endotoxins detection - from Limulus amebocyte lysate to recombinant factor C. Chapters 10 and 11 discuss the role of endotoxins in infection in case of
Helicobacter pylori and Campylobacter jejuni, and also the role of Pseudomonas LPS in cystic fibrosis airway infection. Chapters 12-14 consist of data about development of small-molecule endotoxin agents, anti-endotoxin vaccine for sepsis, and synthetic and natural TLR4 agonists as safe and effective vaccine adjuvants.

Chapter 15 deals with targeting endotoxins in the treatment of sepsis. Chapters 16 and 17 highlight LPS in Rhizobium-legume symbioses and in plant innate immunity.

The book is well prepared. There are many good quality photos, graphs, and pictures. Each chapter ends with a base reference, and there is a subject index at the end of the book.

The book will be very useful for many researchers in the field of biochemistry, biotechnology, immunology, and medicinal chemistry. This book can also be a useful tool for teachers and students of colleges and universities. 